\title{
A Method for Autonomous Positioning Avatars in a Group
}

\author{
Fons Kuijk \\ Distributed and Interactive Systems \\ CWI \\ Amsterdam, Netherlands \\ Fons.Kuijk@cwi.nl
}

\begin{abstract}
In this paper, we describe a method to position a group of avatars in a virtual environment. The method aims at a group setting that seems natural for a group of people attending a guided tour and was developed in particular to assist participants by autonomously positioning their avatars on each stop of a virtual tour. The geometry of the virtual environment is key input, but also engagement of participants and possible social networks are taken into account. Consequently, it may serve to position avatars in similar type of situations.
\end{abstract}

Keywords - affect; educational trip; engagement; navigation; virtual environment; positioning; social aware

\section{INTRODUCTION}

Teachers can take their students on educational trips, or field trips, for instance to visit, zoos, theaters, science or art museums or historical sites. By actually bringing them to these sites the students are more likely to retain information, and to understand and appreciate the importance and relevance of what they are learning [1] [2]. Financial cost, lack of time, safety and travelling distance may make it difficult to organize educational enriching trips. These difficulties can be avoided by shifting the real to the virtual. We created a generalpurpose tele-immersion system, REVERIE, that enables individuals, represented by virtual characters, to interact in a shared 3D synthesized environment. This tele-immersion system shows participants what can be seen in a virtual environment from the dynamically changing point of view of their virtual representation. 3D spatial audio that enables personal conversations with other participants completes the impression of being in an as-real environment. As such this general-purpose system is well suited for a virtual educational trip, preparing for a real trip — since that will improve on-task behavior [3] — , or even to replace the real trip altogether.

A teacher can take his class on a virtual educational trip in one of the virtual environments we produced (a gallery and a historical site). The students are free to explore these environments or may be given worksheets to instruct them, but the system can also provide an autonomous agent to take them along on an organized guided tour. The autonomous agent acting as virtual tour guide typically moves from one place to another. The students are supposed to follow this tour guide by navigating in the virtual environment. Students may be familiarized with manual navigation in a virtual environment, but even then, it can be tedious as it does not come as natural as walking in a real environment. Hence for user comfort our system can assist by taking care of

REVERIE is funded by the European Community's Seventh Framework Programme (FP7/2007-2013) under grant agreement no. ICT-2011-7-28772 repositioning the students' avatars at each stage of the tour. The avatars are made to follow the tour guide automatically and are positioned near the place where the tour guide stops to continue his narrative. This does not require any action of the students (though, if they prefer to do so, they can step in to take control at any time).

In this paper, we describe our method for autonomous positioning avatars of a group of students that considers geometry of the environment, student engagement and social networks to result in a setting that seems natural for a group of students attending a guided tour. Related work is presented in section II. Section III describes the method of autonomous positioning and section IV presents results and conclusions.

\section{RELATED WORK}

A group of entities moving through space can be described by a model known as flocking [4], a model that relies on a few rules on separation, alignment and cohesion leading to realistic movements of the individual species of a coherent group. Similar aspects of this model can be found in simulations of a crowd as in [5], [6] and [7]. These models focus on the dynamics of the movements rather than on final positioning. In [8] we find a method to provide goal locations maintaining the personal space of non-related individuals, and [9] is a framework on scalable and adaptive group formation. However, these all do not provide the specific result we are looking for: avatars of each participant should navigate towards a position that provides a clear view on the tour guide, not hindered by obstacles and preferably reflects engagement and social network of the participants.

\section{A METHOD FOR AUTONOMOUS POSITIONING}

For navigation, our system makes use of path planning and obstacle avoidance software based on Explicit Corridors [10]. This navigation software provides collision free walks from a character's current position in space towards a target position. It can handle a virtually unlimited number of characters moving at the same time and allows individual settings of characteristics as walking speed, clearance, side preference and interpersonal distance [11].

An interactive tour guide is programmed to move from one position to the next to give an explanation on points of interest based on a script that describes all aspects of the excursion. The guide stops at positions that are chosen with care, so that the guide can point at points of interest and is oriented towards an empty area where followers can position themselves to have a clear view on both the guide and the points of interest.

Given this, to obtain autonomous positioning of avatars we developed a method to assign appropriate target positions to 
these avatars for each point where the tour guide stops. The navigation software is then instructed to bring them there.

Autonomous positioning is triggered when the tour guide receives an instruction to navigate towards the next location. The method involves the following steps:

A. Generate candidate positions;

B. Assign positions to avatars;

C. Initiate navigation.

Next these steps are described in more detail.

\section{A. Generate candidate positions}

An algorithm generates as many candidate positions as there are avatars participating in the tour. The algorithm involves the five phases described below.

\section{1) Basic positions}

Assuming the tour guide is positioned in a rational way, as stated above, we take position and orientation of the tour guide as basis for candidate positions of the followers. As people following a tour tend to position themselves in front of a tour guide, we start by taking candidate positions to lie on circular sections in front of the guide as illustrated in Figure 1. These circular sections span an angular range a human being's gaze can reach comfortably. In accordance with the observation that interpersonal distance in a virtual environment relates to gaze [12] the figure shows that the interpersonal distance between the guide and the followers on the first row is taken to be bigger than the interpersonal distance between the followers themselves, since all followers are positioned to face the guide.

These two parameters, the interpersonal distances and the angular span, determine the first step of the algorithm that generates initial candidate positions: a vector of length of the facing interpersonal distance from the guide forward produces the first candidate position. This vector is rotated to obtain a second position flanking the first position; the third position is obtained by rotating the vector in the opposite direction. By starting in front of the guide and alternately adding positions at both sides until the angular span is covered, the first row is filled. For each next row, the length of the vector is increased and the same procedure is repeated. The length increase of the vector and the angular rotation for each row is such that the distance between candidate positions equals the interpersonal distance of non-facing characters. For each even row the initial vector is rotated half the rotation angle to avoid the center positions of all rows to be lined up.

\section{2) Adding irregularity}

From Figure 1 it is obvious that the result of the first phase is too regular to reflect what people are inclined to do. Hence, we introduce two options to add irregularity.

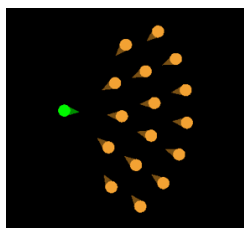

Fig. 1. Initial candidate positions of the followers (orange) lie on a circular grid-like structure around the tour guide (green).
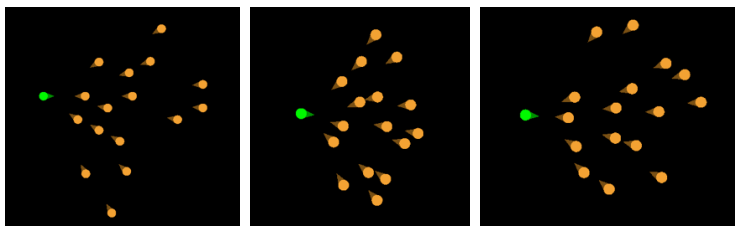

Fig. 2. Adding irregularity by skipping positions (left), randomizing positions (middle), or both (right).

One option is to randomly skip positions, increasing the chance of skipping at each next row, starting for instance from a $10 \%$ chance at the first row. The noticeably less compact result is shown in Figure 2 on the left. The positions may still be on a regular structure, yet due to the open spaces created it becomes less obvious.

Alternatively, positions can be randomized by adding a small random distance shown in Figure 2 in the middle. The interpersonal distance is increased in relation with the maximum random distance added to adhere to the rules of preferred interpersonal distance [11] [12].

A combination of both options is shown in Figure 2 on the right. The (ir)regularity and compactness of the group can be varied by altering the chance of skipping positions and the maximum random distance added.

\section{3) Check for accessibility}

The above phases did not deal with obstacles and did assume there was sufficient space to position all followers. In general, space is limited and can be full of obstacles. So, each candidate position has to be checked for accessibility.

The navigation software can verify accessibility on three aspects. First it verifies that the candidate position is not on top of an obstacle. If this is not the case it checks that the position is at sufficient distance of obstacles (as defined by a clearance parameter). Lastly, it is verified that walking from the current position to this candidate position without being blocked by obstacles is possible. If one of these checks fails the candidate position is rejected.

When the accessibility check fails, the chance of skipping positions is reset to zero; the motivation to do so is that space may be in short supply and should be used more efficiently. Yet, for each next row the chance of skipping will increment as before, provided there are no obstacles on that row.

As an example, Figure 3 shows a situation with inaccessible candidate positions that are rejected. The tour guide is positioned near to a wall shown on the right-hand side and facing it. As a result, the amount of space to position the followers in front of the tour guide is limited. For the first couple of rows, generation of candidate positions starts as normal with both randomization of positions and skipping of

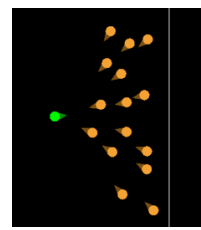

Fig. 3. If space is limited due to obstacles, as here a wall on the righthand side, the method leads to a more compact setting. 
positions. The accessibility check does not lead to rejection yet, so skipping positions remains enabled. Near to the wall however, accessibility fails, so skipping is cancelled, leading to a more compact setting as compared to the setting of Figure 2 on the right.

\section{4) Check for visibility}

Followers should have a clear view on the tour guide, that is, they should not be positioned behind an obstacle. For this the method includes an occlusion test. A ray cast between the follower's head position and the tour guide's head position should not hit a bounding box of static objects. If it does so, the candidate position is rejected and, similar to the accessibility test, the chance of skipping candidate positions is reset to zero. This occlusion test does not include occlusion by avatars, since these are dynamic and can change position. In our system users can take a small step sideways just by hitting a key if needed. In Figure 4 it shows that candidate positions behind obstacles that block visibility (shown as white objects) are rejected, whereas those behind nonblocking objects (shown as grey objects) are not (see the topmost position from which the follower can look over a nonblocking object and still see the tour guide's face).

\section{5) Handle lack of space}

In the above examples, there was sufficient space to position 16 followers in the preferred area in front of the tour guide. If the tour guide would be closer to the wall and the space in front of the tour guide is more confined, or if there would be more followers, this may no longer be the case. The area to place followers is hard limited by the wall and soft limited by the angular span. To be able to add more followers in a confined space like that, we first make use of the positions that were skipped in the second phase to increase irregularity. The algorithm maintains a list of all positions that were skipped. Note: these skipped positions are not those that are rejected on basis of accessibility and visibility and therefore can still be added to the set of candidate positions. In this way, the setting is made as compact as possible given the average interpersonal distance and available space.

Adding the skipped positions may not be sufficient. In the example of Figure 5 on the left, the tour guide is near a corner facing a wall. The preferred area in front of the tour guide is not enough space to position all followers, even using the initially skipped positions. In this situation, we also allow followers to be positioned beyond the angular span in front of the tour guide. The algorithm starts filling the rest of the space around the tour guide, first extending the span of the first row until it covers a full circle, then likewise for the next rows until all followers have obtained a position. We decided not to skip

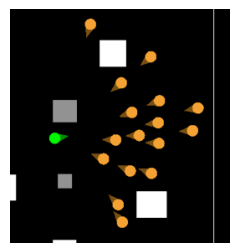

Fig. 4. The method accounts for obstacles in the virtual space that block the line of sight (white) and those below the line of sight (grey). positions for candidate positions behind the tour guide, and only randomize the positions, though there is no strong argument to do so. No skipping implies that the average interpersonal distance remains constant, in this way maintaining the most compact acceptable setting. Obviously, based on accessibility and visibility checks positions still may be rejected, but at the limit the entire virtual environment that is accessible and from which a clear view on the tour guide is assured can be used to position followers. When we try to squeeze in more avatars than can be handled in this way, the virtual environment can no longer provide the required personal space for all participants.

\section{B. Assign positions to avatars}

Once all candidate positions for the followers have been generated, we assign these positions to the avatars. People in real life will have a strategy to position themselves with respect to a tour guide. Those enthusiastic and interested in the presentation may try to get upfront to make sure they will not miss a word of the presentation, while timid people or those less interested will put themselves more at the back of the group. Also, relatives and friends are likely to team up. These two factors, engagement and social relations, help position avatars on a human-like strategy.

We apply a simple approach to uncover social relations between participants. When participants are free to move around and one of them navigates towards another participant's avatar we assume that these two have a social relation, as getting close to each other in the virtual environment is needed to have a private conversation. We "connect" a participant to its most recent partner based on this assumption. More advanced methods can be found in [13] or [14]. Couples that we believe to have a social relation will be treated as one entity for placement, and the avatars of this couple will be assigned to adjacent places.

The definite placement of avatars relates to engagement. In our system engagement is inferred from the gaze of the user but, depending on the equipment used, alternatives as can be found in [15] and [16] may do as well. Based on the level of engagement the strategy to assign positions to avatars is simple: avatars of the most engaged participants get the best upfront positions, avatars of the least engaged participants will be placed on more distant positions. For social couples, we use the average level of engagement. Best places are those in front of the tour guide, each next row is considered to be of somewhat lower quality and the positions behind the tour guide are considered to be the worst.
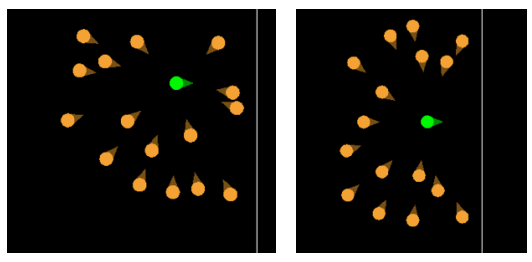

Fig. 5. When the most compact setting in front of the tour guide cannot offer sufficient positions for the followers the algorithm will start to position followers behind the tour guide. 
Note, the above strategy for positioning avatars of students can be of use for a teacher observing the students, because students that may need encouragement - or a reprimand can easily be found at the back of the group.

\section{Initiate navigation}

The last step of the method is to make the avatars navigate towards the positions that have been assigned. The instruction for the tour guide agent to navigate to the next location of the tour is leading. This instruction triggered the process of autonomous positioning at the first place and it makes sense that the tour guide is the first to start to move. Next, all avatars will be put in action by an instruction that includes the position assigned to them, the orientation they have to turn to when they arrive at that position (directed towards the tour guide), the preferred walking speed and the time they can start the navigation. The navigation software of our system receives these instructions and provides collision free walks to the positions as specified.

The preferred walking speed and the timing of the navigation has been made to relate to the level of engagement. Avatars of the most engaged participants will be the first to start moving, shortly after the tour guide starts to move. These avatars will walk at roughly the same speed as the tour guide. The avatars of less engaged participants will start moving somewhat later and will walk at a lower speed. In this way avatars of the most engaged participants will be the first to arrive at the new location, which makes sense, since they will be put upfront anyway. Avatars of the least engaged participants will be the last to arrive, which goes well with the fact that they were destined to end up at the back of the group.

\section{RESUltS ANS CONCLUSIONS}

The system has been evaluated based on a guided tour in a cultural education setting (see Figure 6). Before the autonomous positioning was available, navigation was done using map navigation (clicking on a floorplan image) and keyboard only. Then nine out of ten users indicated having problems taking a position in the virtual environment (e.g., to obtain a proper group formation relative to others). Once the autonomous positioning was included this issue was no longer reported. The capabilities of the method on very large groups have been demonstrated in simulations, but practical issues made it impossible to verify how participants in larger settings will value it.

We have presented a method for autonomous positioning of avatars. The method generates positions that human beings in a guided tour could opt for as well, first using the available space in front of the tour guide, if that is insufficient filling up the space around the tour guide, at maximum filling the complete virtual environment. All positions are guaranteed to have a view on the tour guide not obstructed by static objects. The assignment of positions in the virtual environment relates to engagement of the participants. Getting the best upfront positions rewards the more engaged participants. Buddies that have a social relation get a position next to each other. Designed for avatars participating in a guided tour in a virtual environment, the method may be an inspiration for similar applications where social aware positioning of avatars relative to a location may be needed.

\section{REFERENCES}

[1] Kulas, Michelle. (2014) "What are the Benefits of Field Trips for Children?". LIVESTRONG.com. January 2014

[2] Greene, Jay P. Kisida, Brian. Bowen, Daniel H. (2014), "The Educational Value of Field Trips".In Education Next. Vol 14, no 1

[3] Bitgood, Stephen. (1989), "School Field Trips: An Overview" in Visitor Behavior (1989) 4(2) 3-7

[4] Reynolds, Craig W. (1987). "Flocks, herds and schools: A distributed behavioral model." ACM SIGGRAPH Computer Graphics 21 (4). pp. 25-34.

[5] Dyer, John R.G. Ioannou, Christos C. Morrell, Lesley J. Croft, Darren P. Couzin, Iain D. Waters, Dean A. Krause, Jens (2007) Consensus decision making in human crowds. In Animal Behaviour, Vol 75, No 2, pp 461-470

[6] Pelechano N., Allbeck, J., Badler N. I. (2007): Controlling Individual Agents in High-Density Crowd Simulation. In $A C M$ SIGGRAPH/Eurographics Symposium on Computer Animation (SCA), (August 2007), 99-108.

[7] Thalmann, Daniel. Musse, Soraia Raupp (2013), Crowd Simulation. ISBN $9781447144502 \cdot 9781447144496$

[8] Li, W. Di, Z. Allbeck, J. "Crowd distribution and location preference", in Computer Animation and Virtual Worlds 23(3-4) · May 2012

[9] Gu, Q. Deng, Z, "Formation Sketching: An Approach to Stylize Groups in Crowd Simulation" in Proceedings - Graphics Interface · January 2011

[10] Geraerts, R., (2010), Planning Short Paths with Clearance using Explicit Corridors. In IEEE International Conference on Robotics and Automation, pp. 1997-2004

[11] Hall, E.T. (1973), The Hidden Dimension, in Leonardo, 6(1), 94

[12] Bailenson JN(1), Blascovich J, Beall AC, Loomis JM. (2003), Interpersonal distance in immersive virtual environments. In Pers Soc Psychol Bull. 2003 Jul; 29(7):819-33.

[13] Kappe, Frank. Zaka, Bilal. Steurer, Michael (2009), Automatically Detecting Points of Interest and Social Networks from Tracking Positions of Avatars in a Virtual World, In International Conference on Advances in Social Network Analysis and Mining, 2009. ASONAM '09.

[14] Alletto, S. et al. (2014), From Ego to Nos-vision: Detecting Social Relationships in First-Person Views, In IEEE Conference on Computer Vision and Pattern Recognition Workshops (CVPRW), 2014, pp 594 599, DOI: 10.1109/CVPRW.2014.91

[15] Sanghvi, J. Castellano, G. Leite, I. Pereira, A. McOwan, P W. and Paiva, A. (2011). Automatic analysis of affective postures and body motion to detect engagement with a game companion. In Proceedings of the 6th international conference on Human-robot interaction (HRI 11). ACM, New York, NY, USA, 305-312. http://doi.acm.org/10.1145/1957656.1957781

[16] Baker, R. et al. (2012) Towards Sensor-Free Affect Detection, In 5th International Conference on Educational Data Mining, EDM 2012
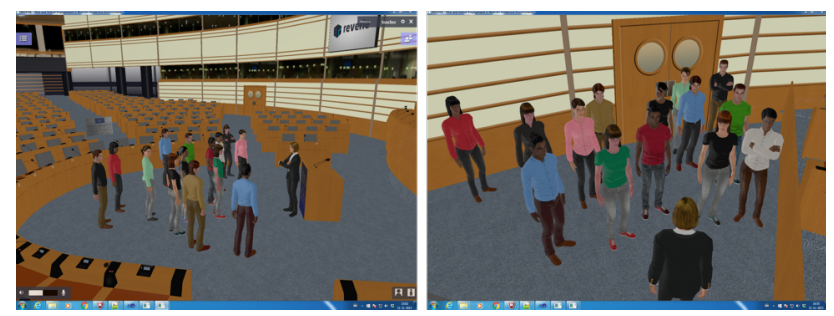

Fig. 6. Left: typical group placement in front of a tour guide, right: a more confined space leads to a more compact setting 